Finisterra, XXXV, 70, 2000, pp. 115-127

\title{
LE PORTUGAL DANS LE LIVRE DE GÉOGRAPHIE POUR LA JEUNESSE AU XVIIIe SIÈCLE ET AU DÉBUT DU XIXe
}

BERNARd HubER 1

Résumé - Il s'agit d'une réalité peu connue: dès le XVIIIe siècle, une infime partie de la jeunesse de la plupart des pays européens avait à sa disposition nombre d'ouvrages relatifs à la géographie. Quelle place le Portugal occupait-il dans cette littérature? Quelle image de ce pays les auteurs, par le texte, voire l'illustration, voulaient-ils donner à l'enfant? Telles sont les questions auxquelles le présent article tente d'apporter des éléments de réponse.

Mots clés: Portugal, XVIII et XIXe siècles, littérature géographique, jeunesse.

Resumo - Portugal nos livros de geografia Para a JUVEntude no SÉculo XVIII E NO INÍ́cIO DO SÉcUlo XIX. Trata-se de uma realidade pouco conhecida: desde o século XVIII, uma minoria da juventude da maior parte dos países europeus tinha à sua disposição obras relacionadas com a geografia. Que lugar ocupa Portugal neste tipo de literatura? Qual é a imagem que os autores querem dar às crianças através dos textos e ilustrações? Estas são as perguntas às quais esta nota espera fornecer elementos de resposta.

Palavras-chave: Portugal, séculos XVIII e XIX, literatura geográfica, juventude.

\begin{abstract}
PoRTUGAL IN GEOGRAPHY BOOKS FOR THE YOUNG IN THE 18TH CENTURY AND EARLY 19TH CENTURY. It is a little known fact but since the 18th century a small number of young people in most European countries have had access to many books on geography. What is Portugal's position in this type of literature? What image do the authors want to give children through their texts and illustrations? This note seeks to find answers to these questions.
\end{abstract}

Key words: Portugal, 18th and 19th centuries, Geography Books, young people.

1 Chargé d'enseignement, Faculté de Psychologie et des Sciences de l'Éducation, Université de Genève, 9, route de Drize, 1227 Carouge, Suisse; E-mail: bernard.huber@pse.unige.ch 


\section{INTRODUCTION}

Le public l'ignore généralement: dès le XVIIIe siècle, rarement avant, de nombreux ouvrages de géographie existent à l'intention de la jeunesse noble et bourgeoise: abécédaires géographiques, abrégés de géographie, atlas, entre autres.

Le Portugal, naturellement, y occupe une place. Laquelle? Comment les auteurs présentent-ils ce pays à l'enfant?

C'est sur ces questions que nous désirons nous pencher dans les lignes qui suivent. Ces dernières sont étayées par l'étude attentive de nombreux livres d'enfant, anciens et rares, que nous avons rassemblés depuis douze ans et qui constituent notre fonds privé.

\section{LE LIVRE D'ENFANT: UNE HISTOIRE ANCIENNE}

Si, peu après l'apparition de l'imprimerie à caractères mobiles, au milieu du XVe siècle, les premiers livres destinés à l'enfance sont publiés (bibles, catéchismes et abécédaires principalement), il faudra attendre le XVIII ${ }^{e}$ siècle pour assister à l'émergence d'une véritable littérature géographique destinée à la jeunesse. Cette situation générale souffre naturellement quelques exceptions. Ainsi, F. Buisson (1968:189) évoque l'existence d'un ouvrage de ce type, imprimé à Genève en 1580 déjà, destiné aux enfants du patriciat.

À la fin du XVIII e siècle ces ouvrages font florès en Angleterre, en France et dans les pays germaniques principalement. La bibliographie de H. Marques Jr (1928), consacrée aux livres d'enfant portugais publiés de 1603 à 1927 ne nous indique en revanche aucun ouvrage de ce type. Mais ce répertoire, comme tout autre, ne peut prétendre à l'exhaustivité. Quoi qu'il en soit, l'auteur reconnaît que le Portugal n'a jamais été un pays phare dans le domaine de la littérature pour la jeunesse. L'analphabétisme ( $80 \%$ de la population environ en 1890) qui, ne l'oublions pas, n'a été éradiqué que relativement récemment, est la principale cause de cette situation. M. L. Bettencourt Pires, dans son travail, n'est guère plus loquace à cet égard. Néanmoins elle souligne le fait (p. 35) que les échanges consécutifs aux voyages au long cours qu'ont effectués les navires portugais en Afrique, en Amérique, en Asie et en Océanie, ont contribué à enrichir une littérature orale dont les enfants, à quelque époque que ce soit, ont toujours été friands.

Ce manque d'information relativement à cette question ne signifie toutefois pas qu'il n'y ait pas eu d'ouvrages de ce genre au Portugal. Il y en a eu, c'est plus que probable; mais nous ne les avons jamais vus. Destinés à un public des plus restreints, ces volumes ne font l'objet, la plupart du temps, que de tirages limités. Il en résulte que les exemplaires qui sont parvenus jusqu'à nous, qui ont survécu aux fléaux menaçant tout livre (feu, humidité, lumière, insectes, notamment), sont rares. Ils le sont d'autant plus que, chacun le sait, 
les enfants, quelle que soit l'époque, n'ont pas pour qualité première de prendre soin des livres qu'ils ont entre leurs mains.

\section{LE PORTUGAL AU XVIIIe SIÈCLE}

Avant d'entrer dans le vif du sujet il convient de brosser un tableau politique, économique et social du Portugal à l'époque des Lumières et au début du XIXe siècle.

L'apogée de ce pays est révolue depuis longtemps. Les grandes découvertes des XVe et XVIe siècles appartiennent au passé. Le Portugal du XVIIIe siècle vit de ses conquêtes antérieures, du Brésil notamment. Dès le XVIIe siècle il est concurrencé par des pays qui émergent, qui s'affirment et qui, parfois, prennent sa place. C'est le cas, par exemple, des Pays-Bas dans les îles de la Sonde (Timor). L'immense empire lusitanien, difficile à contrôler pour une métropole aux moyens humains et pécuniaires modestes, commence progressivement à se lézarder. Le traité luso-anglais de Methuen, signé en 1703, met également en évidence la faiblesse croissante du Portugal. Le faste du règne de Jean V est largement artificiel. L'or du Brésil, qui lui permet la construction du palais de Mafra et celle de la splendide bibliothèque de Coimbra, notamment, cache mal une autre réalité: la pauvreté de la population et le sous-équipement général du pays (voies de communication mauvaises ou inexistantes, manufactures sous-développées, etc.). Dans la deuxième moitié du XVIIIe siècle, cet or permettra encore au marquis de Pombal, ministre de Joseph Ier, de reconstruire Lisbonne après le tremblement de terre de 1755 et d'entreprendre de grands travaux d'utilité publique. Ces investissements ne constituent toutefois qu'une embellie. Le pays s'affaiblit inexorablement. C'est au point que, pendant une douzaine d'années, au début du XIXe siècle, l'Angleterre domine complètement le Portugal. Enfin, l'indépendance du Brésil, proclamée en 1822, n'est évidemment pas sans conséquences sur la métropole.

Pour clore avec le contexte général, nous pouvons dire qu'à cette époque le Portugal se trouve dans une phase de décadence. Ses dirigeants tentent de surseoir à cette funeste situation. Cette dernière est, pour les raisons évoquées, inéluctable.

\section{DU LIVRE D'ADULTE ADAPTÉ À L'ENFANCE AU LIVRE D'ENFANT PROPREMENT DIT}

Pendant la plus grande partie du XVIIIe siècle, les ouvrages de géographie destinés à la jeunesse ne sont le plus souvent que des abrégés de textes destinés à l'adulte. Ils ne se distinguent en rien des autres ouvrages, aussi bien sur le plan de leur présentation extérieure, qui suit les canons alors en vigueur pour tout livre, que sur celui des contenus. L'enfant est encore perçu comme un «adulte incomplet». Ce n'est que vers l'extrême fin du XVIII ${ }^{\mathrm{e}}$ siècle, suite à 
la diffusion des idées émises par J.-J. Rousseau dans Émile ou de l'éducation, que, désormais, il est considéré comme un être sui generis et, partant, dispose de livres dont le contenant (apparence extérieure) et le contenu (texte, illustration, couleur, etc.) tiennent compte des idées nouvelles.

\section{LA PLACE DU PORTUGAL DANS CETTE LITTÉRATURE}

\section{Le texte}

La phase de régression que connaît le Portugal a une incidence directe sur la place relativement modeste qu'occupe ce pays dans la littérature géographique pour la jeunesse de cette époque, si l'on considère le nombre de pages et d'illustrations. Mais, en raison de ses possessions d'outre-mer, on retrouve ce pays dans les chapitres consacrés à l'Afrique, à l'Amérique, à l'Asie et à l'Océanie.

D’une manière générale, les pages consacrées au Portugal précèdent ou suivent immédiatement celles relatives à l'Espagne. Parfois, ces deux États sont traités ensemble, pour certains de leurs aspects tout au moins. À propos de la péninsule ibérique, nous lisons par exemple: «Deux royaumes partagent, quoique fort inégalement, cette péninsule: celui d'Espagne occupe presque les cinq sixièmes de sa surface; le Portugal, resserré à l'ouest sur l'Atlantique, paraît, par son peu d'étendue, n'être qu'une province du premier. Aussi, dans la description des accidens géographiques qu'offre la péninsule... ne nous astreindrons-nous pas à la division politique de ces deux états...» (BAILLEUL et al, 1826: 598) 2.

Le Portugal est très souvent comparé à son voisin oriental. Il est véritablement mesuré à l'aune de ce dernier : «L'Espagne est bien plus considérable que le Portugal» (Mentelle, 1781: 293). Il en est de même de sa population. Le lecteur est immanquablement frappé par ce rôle d' «appendice de l'Espagne» que les auteurs assignent généralement au Portugal. Cette situation apparaît très clairement dans une très rare géographie napolitaine dans laquelle le Portugal est inclus dans le chapitre XI, relatif à l'Espagne. Les lignes que consacre au Portugal A. M. Cinno (1795), son auteur, n'apparaissent-elles pas, en effet, sous le sous-titre «Appendice del Portogallo» (p. 56)? Est-ce là une conséquence d'un lointain passé commun? Le principal manuel scolaire suisse romand de géographie de la fin du XVIIIe siècle et du début du XIXe dit à ce propos : «Le Portugal dépendoit autrefois de l'Espagne. Il a eu pendant long-tems le même sort que ce royaume, et n'a commencé qu'au onzième siècle à former un état séparé.»(OsTERVALD, 1813: 253). Est-ce peut-

2 Dans le présent texte, l'orthographe ancienne est respectée. 
être une réminiscence de l'occupation espagnole entre les années 1580 et 1640 ? Ce sont des hypothèses. Cette réalité est d'autant plus singulière que, chacun le sait, le Portugal a toujours cherché, au cours de son histoire, à se démarquer de son puissant voisin. De tout temps, Lisbonne a tourné ostensiblement le dos à Madrid. La traditionnelle faiblesse des voies de communication terrestres reliant les deux pays, routières d'abord, ferroviaires ensuite, qui, d'ailleurs, prévaut encore aujourd'hui, n'est qu'une illustration parmi d'autres de cette situation.

\section{L'iconographie}

Quelques cas exceptés, dont l'Orbis pictus de Coménius (1658), qui est l'un des premiers livres illustrés pour la jeunesse, l'image dans le livre d'enfant ne commence réellement à se répandre que vers la fin du XVIII e siècle. Mis à part une carte du Portugal insérée dans l'Atlas des enfans, ou nouvelle méthode pour apprendre la géographie, nous n'avons pas trouvé d'illustration relative à ce pays dans les nombreux livres de géographie pour la jeunesse du XVIIIe siècle que nous avons lus. En revanche, ce pays est représenté, certes modestement, au siècle suivant. Dans les ouvrages qui constituent notre fonds, le Portugal est illustré une fois par une corrida, l'autre fois par le port de Lisbonne, "... un des plus beaux mouillages de l'Europe.»(SAINTES, 1841: 78).

\section{Thèmes principaux abordés}

Les textes concernent aussi bien la métropole que les colonies. Pour un pays comme le Portugal, il n'est pas possible de parler de la première sans évoquer les secondes.

Les ouvrages ne proposent, en fait, que de simples descriptions, les auteurs se bornant à énoncer des faits. Ils ne cherchent pas à susciter la réflexion de l'enfant. Ils adoptent le plus souvent la méthode catéchétique, faite de questions et de réponses alternées, ces dernières devant être apprises par cœur. Cette situation est normale pendant la plus grande partie du siècle des Lumières, étant donné les conceptions que l'on avait à cette époque de l'éducation en général et de l'enseignement de la géographie en particulier. Elle perdure assez longtemps, même après la diffusion des idées relatives à l'enseignement de cette discipline énoncées par J.-J. Rousseau dans le livre troisième de Émile ou de l'éducation et mises en œuvre, à la fin du XVIII ${ }^{e}$ siècle, par les philanthropes allemands ainsi que par H. Pestalozzi et ses disciples - K. Ritter, le rénovateur de la géographie, entre autres - au début du siècle suivant.

Quels sont les thèmes principaux abordés?

Tous les ouvrages, quoique ne suivant pas nécessairement le même plan, évoquent habituellement les éléments ci-après: situation géographique générale de la métropole, hydrographie, orographie, climat, population, économie 
120

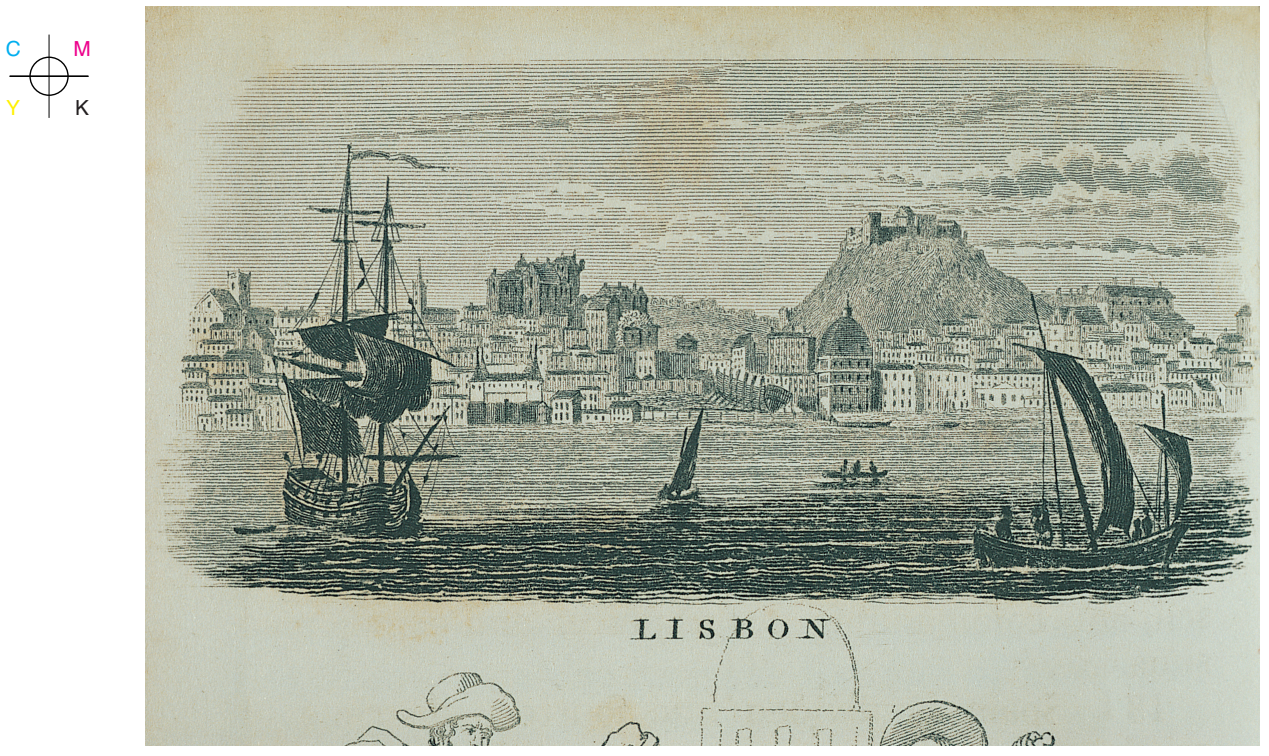

Fig. 1 - «Lisbon» (Goldsmith, 1834)

Fig. 1 - «Lisboa» (Goldsmith, 1834)

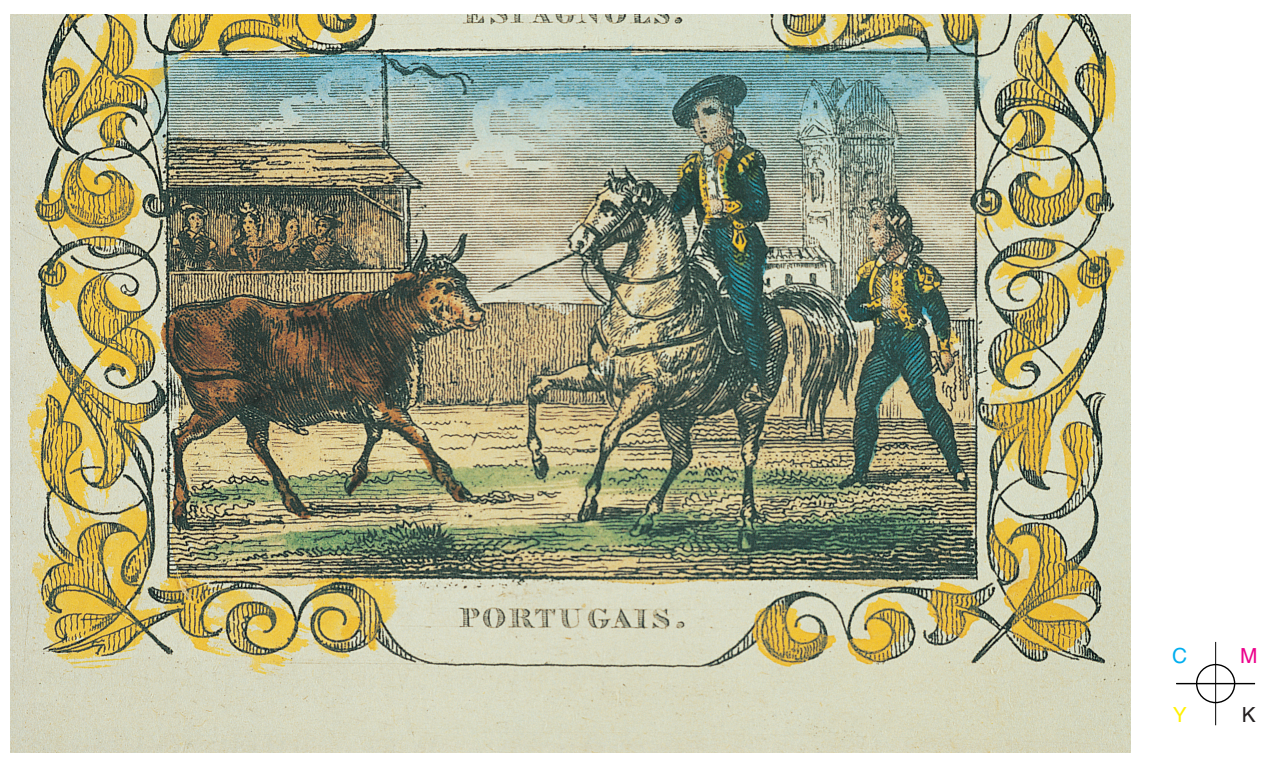

Fig. 2 - «Portugais» (Saintes, 1841)

Fig. 2 - «Portugueses» (Saintes, 1841) 
(ressources naturelles, productions, échanges, etc.), histoire, culture et, enfin, colonies. C'est donc une géographie «à tiroirs», si décriée par la suite, qui est proposée.

Quels sont donc les principaux points qui apparaissent dans chaque rubrique?

a) Situation géographique

En ce qui concerne la situation géographique générale de la métropole, les chapitres relatifs au Portugal commencent par évoquer la position du pays par rapport à son voisin et à l'océan atlantique. Ils sont souvent subdivisés en paragraphes correspondant aux diverses provinces. C'est à l'intérieur de ces subdivisions que l'on trouve certains éléments du plan que nous évoquons ci-dessus.

b) Hydrographie et orographie

Pour ce qui a trait à l'hydrographie, ce n'est pas l'incontournable liste onomastique des principaux cours d'eau qui nous frappe, car elle est présente dans toute description de pays, mais l'évocation de nombreuses sources thermales, de la qualité des eaux minérales qu'on y trouve et, enfin, de l'excellence des ports portugais, souvent situés dans des estuaires parfaitement abrités.

Rien de particulier ne caractérise l'orographie du pays dans ces ouvrages, qui se contentent d'évoquer le nom de certains reliefs.

c) Climat

La question du climat est largement développée. Il est tellement favorable, si l'on en croit ces textes, que le Portugal est un véritable pays de cocagne. Il s'ensuit nous dit-on, que ses productions agricoles sont riches et variées. L'air y serait pur, sain, doux et tempéré car sous l'influence océanique, ce qui n'est pas le cas, précise-t-on, des provinces espagnoles situées aux mêmes latitudes mais à l'intérieur des terres. Un manuel suisse romand de géographie dit à ce propos : "Le Portugal, très favorisé de la nature, jouit d'une température douce et agréable. le voisinage de la mer, joint à l'élévation d'un sol montueux, tempère l'ardeur des rayons de soleil. Des pluies fréquentes rendent l'hiver assez. incommode dans les provinces du nord; mais rien de plus beau et de plus enchanteur que le printemps en Portugal.» (Cours de géographie ancienne et moderne, suivi des élémens de l'astronomie, 1830:179).

\section{d) Population}

Ce qui a trait à la population, est habituellement généreusement abordé. Outre les données factuelles banales relatives au nombre d'habitants et aux villes principales qui figurent entre autres dans ces ouvrages, s'y trouvent des 
éléments bien plus intéressants concernant les traits physiques et de caractère d'une part et les mœurs des Portugais d'autre part. Les stéréotypes, souvent outranciers, sont omniprésents. Dans ce type de littérature, il est opportun de le souligner, il en est ainsi de toute population. Aucune n'y échappe en effet. Relevons par exemple que les Islandais «... passent en général pour un peuple ignorant et superstitieux...» (GoRDON, 1748:176), que les Arabes sont considérés comme des voleurs de grands chemins, des gens impudiques et cruels (id.: 251-254) et que les Guinéens seraient des personnes lâches et sales (id.: 261). Les Espagnols, malgré leurs qualités souvent relevées, seraient «... universellement négligens, fort adonnés aux femmes, jaloux jusqu'à l'excès...» (id.: 110). Des passages marquants concernant les Portugais, nous avons extrait le suivant : «Les Portugais autrefois si renommés pour leur science de la navigation, et pour les vastes découvertes, dont le monde leur est redevable, ont bien dégénéré de la vertu de leurs ancêtres. Quelques auteurs ont pris plaisir à les caractériser ainsi : prenez un de leurs voisins (un naturel Espagnol) retranchez-en toutes les bonnes qualités, ce qui est bientôt fait; ce qui reste composera un Portugais complet. On regarde en général les Portugais comme des gens traîtres les uns envers les autres, mais surtout envers les étrangers; extraordinairement rusés dans leurs procédés, adonnés à l'avarice et à l'usure, cruels jusqu'à la barbarie quand ils ont la supériorité; le petit peuple parmi eux est universellement porté au larcin; indépendamment de toutes ces qualités on prétend que ce peuple est fort méchant, et que c'est un reste du sang des Juifs mêlé avec celui des Portugais.» (id.: 113)... Ces lignes, rien de plus normal, étonnent, voire choquent. C'est là néanmoins le tableau qui était brossé à certains enfants anglais et français, au XVIII ${ }^{\text {e }}$ siècle. En effet, ce passage est tiré d'un ouvrage anglais, explicitement destiné à la jeunesse, et traduit dans la langue de Molière.

Extraites d'un ouvrage français plus tardif, dédié à la prime enfance, les lignes qui suivent, second exemple, insistent davantage sur les traits physiques et certaines habitudes alimentaires : "Les Portugais sont moins grands que les Espagnols. Ils ont les yeux noirs, le teint olivâtre, le nez retroussé, les lèvres épaisses. Leur politesse est cérémonieuse, leur esprit prompt, leur bravoure très-grande; mais ils sont jaloux, emportés, vindicatifs. La noblesse est fière et fastueuse. Les paysans, très-pauvres, n'ont presque d'autre nourriture que du pain de maïs et de l'ail.» (Petite géographie vivante, 1845: 22).

D'une manière générale, les adjectifs utilisés le plus fréquemment pour qualifier les Portugais sont les suivants: polis, généreux, fiers, vindicatifs, sombres, graves, sérieux, orgueilleux et «...faineans...»(BouRdon, 1744: 111).

Les exemples ci-dessus le démontrent : quel que soit le peuple décrit, la caricature sombre dans l'outrance. On passe allégrement des traits physiques («nez retroussé») aux traits moraux («vindicatifs»), en passant par des caractéristiques sociales comme, par exemple, les habitudes alimentaires («pain de maïs», «ail»). 
On insiste enfin très fréquemment sur l'incomparable expérience et l'habileté des Portugais dans les domaines de la navigation hauturière et du négoce.

Ces descriptions, il ne faut pas l'oublier, ne sont que le reflet des contextes sociaux, politiques, scientifiques, culturels, religieux et autres, prévalant à l'époque considérée.

e) Économie

Les questions économiques, elles, occupent une place assez importante. Les thèmes principaux abordés sont les ressources naturelles, les productions agricoles et les échanges commerciaux.

La plupart de ces textes soulignent la richesse et la variété du sol portugais: «Le Portugal est un beau pays, riche, fertile et abondant en tout ce qui est nécessaire aux délices de la vie...» (SEPP, 1783: 42). Cette vision idyllique des choses est parfois tempérée. Plusieurs ouvrages signalent en effet le fait que l'agriculture est souvent négligée et que, partant, une grande partie du territoire portugais est en friche. Qu'y trouve-t-on, si on les en croit? Du plomb, du fer, de l'alun, de l'étain, de l'or, de l'argent, du sel, du marbre, de l'albâtre, des eaux minérales en abondance et des pierres précieuses (améthystes), notamment.

En ce qui concerne les productions agricoles, ces livres soulignent fréquemment le fait que le sol est plus adapté à la culture des fruits qu'à celle des céréales. La vigne est, lit-on, fort répandue et produit des vins de qualité. D'une manière générale, les fruits sont qualifiés d'excellents, les oranges en particulier : "Il n'y a pas plus de cent ans qu'on y a transplanté des orangers de la Chine qui y ont extrêmement multiplié, et se sont répandus du Portugal dans la plupart des royaumes de l'Europe, et surtout au midi de la France.» (SEPP, 1783: 43). Les citrons, les grenades, les amandes, les figues et les olives (huile) n'ont, quant à eux, rien à leur envier. Le Portugal produit des plantes aromatiques dont on compose des essences et des «eaux de senteur». Le blé est peu répandu. Le maïs l'est davantage.

L'élevage produit un bétail de grande qualité. Les chevaux, en particulier, sont renommés. Curieusement, la pêche est peu évoquée.

D'une manière générale ces ouvrages mettent en évidence le fait que l'industrie manufacturière est peu développée au Portugal; le mauvais état des routes complique fortement le transport des marchandises à l'intérieur du pays. Il est souligné en revanche que le commerce extérieur de ce pays est très étendu. Le Portugal exporte vers le reste de l'Europe les productions originaires de ses colonies : perles, diamants bruts et autres pierres précieuses, coton, sucre, tabac, gingembre, indigo, bois du Brésil, peaux, poivre, cannelle et plantes médicinales. Il expédie également certaines de ses productions métropolitaines, en particulier vers la Hollande et l'Angleterre : laines, vins renommés, huiles, sel, anis, raisin, figues, oranges et citrons confits.

Qu'importe le Portugal? Du blé, des métaux, des étoffes de luxe, principalement, nous disent ces textes. 


\section{f) Histoire}

Si la présence arabe au moyen âge, la domination espagnole sur le Portugal sous Philippe II, Philippe III et Philippe IV, et le tremblement de terre de 1755 (ce dernier événement a, à l'époque, marqué profondément toute l'Europe; Voltaire lui-même l'évoque dans l'un de ses poèmes) sont très souvent mentionnés, le fait relatif à l'histoire du Portugal qui apparaît le plus souvent est indubitablement l'évocation de son brillant passé, aux XVe et $\mathrm{XVI}$ e siècles, et de sa décadence, au cours des siècles suivants.

La période d'expansion consécutive aux grandes découvertes est en effet largement développée dans ces ouvrages. Le début de la décadence, quant à lui, est concomitant à la montée en puissance d'autres Etats. Un auteur anglais de livres pour la jeunesse dit à ce propos : «Portugal was once a powerful state, especially by sea; but tyranny and the indolence and superstition of the inhabitants have greatly reduced its importance.» (GoLDSMITH, 1834: 42).

\section{g) Culture}

La question de la langue est souvent évoquée : «Le langage des Portugais est un composé de françois et d'espagnol, mais principalement d'espagnol.» (Gordon, 1748: 114), dit cet auteur. Les lusophones «... parlent un dialecte de la langue espagnole.» affirme cet ouvrage anonyme (La géographie en estampes, 1825: 59). Pour lui, la langue portugaise n'a donc pas le même statut que la langue espagnole, la première étant considérée comme «inférieure» à la seconde. Le mot «dialecte», utilisé ainsi, n'a-t-il pas une indiscutable connotation péjorative? La langue espagnole, elle, n'est jamais considérée comme un dialecte de la langue portugaise.

La question des religions est omniprésente dans ces livres. La seule permise, y lit-on, est la catholique romaine. Tous, ou presque, nous gratifient de la liste exhaustive des archevêchés et évêchés portugais. On signale que de très nombreux Juifs ont été convertis de force (Inquisition) mais continuent de pratiquer leurs rites en cachette. On évoque une certaine tolérance envers les marchands anglais établis au Portugal, nombreux, exerçant publiquement leur religion.

Enfin, les arts et l'éducation sont aussi mis en évidence dans certains ouvrages. Le fameux palais de Mafra, souvent cité, est comparé à l'Escurial, près de Madrid. L'université de Coimbra, l'une des plus anciennes d'Europe, est, elle aussi, fréquemment mentionnée. Il en est de même de sa fameuse bibliothèque.

\section{h) Colonies}

La question des colonies occupe une place importante dans les ouvrages dont nous parlons ici.

La puissance passée du Portugal, l'ancienneté de ses conquêtes ressortent clairement de la lecture des chapitres consacrés à l'Afrique et à l'Asie notam- 
ment. En effet, les côtes de ces continents apparaissent au lecteur enfantin comme constellées de belles villes, bien bâties, et de forteresses inexpugnables. Goa, par exemple, "... est comparable aux plus belles villes de l'Europe.» (Cours de géographie ancienne et moderne suivi des élémens de l'astronomie, 1830: 265). L'intérieur des terres est moins bien décrit car, probablement, moins connu.

Outre la liste des comptoirs coloniaux, éphémères ou durables, que l'on trouve dans ces livres, des textes extrêmement intéressants sur la conquête de ces territoires y figurent également. Ils ont dû, sans nul doute, tenir en haleine les enfants privilégiés qui les ont lus. Nous pensons en particulier aux pages que consacrent E. et J. Verreaux (1832), dans leur Océanie en estampes, à l'arrivée des Portugais, au début du XVI ${ }^{\text {e }}$ siècle, dans cette partie du monde. Ce ne sont que combats entre Européens et roitelets locaux, métissage avec les populations conquises, mœurs étranges dont l'anthropophagie tient sans conteste la vedette, faune et flore extraordinaires, produits inconnus et autres faits inhabituels à la jeunesse européenne : «Les Portugais, après de longs combats, parvinrent de bonne heure à former divers établissemens à Timor. Les Hollandais les leur enlevèrent en 1613 et s'établirent dans la baie de Coupang. Cependant les Portugais ne furent pas entièrement expulsés de cette île, et conservèrent à Lifao un établissement qu'ils transportèrent depuis à Dilly ou Diely un peu plus au nord... Les Portugais, par leur long séjour dans cette île, et en s'alliant avec les peuplades du pays, ont produit une race ou variété métive facile à distinguer de toutes les autres; c'est celle des Portugais noirs ou indigènes, qu'on trouve principalement dans la partie septentrionale de l'île... Mais à Dilly même, à la réserve du gouverneur et d'un très-petit nombre d'autres, les seuls Portugais sont noirs et natifs de l'île.» (Verreaux E. et J., 1832: 109).

A propos du Brésil, de ses autochtones et de leurs mœurs, on trouve, dans un autre ouvrage, les lignes qui suivent : "Autrefois les Portugais n'en parlaient qu'avec effroi, et les désignaient sous le nom général d'anthropophages : on a reconnu depuis que les Indiens du Brésil ont en général un caractère doux, docile et sociable.» (Cours de géographie ancienne et moderne, suivi des élémens de l'astronomie, 1830: 322).

De ces textes ressort enfin le fait que, pendant des siècles, si l'on ne tient

pas compte de ses rapports étroits avec l'Angleterre, le Portugal a vécu en circuit fermé avec ses dépendances.

\section{CONCLUSION}

A quelque époque que ce soit, l'enfant a été attiré par les récits de voyages et les aventures qui leur sont liées. Les descriptions de pays le séduisent. Les cartes, elles aussi, ont de tout temps exercé un attrait indéniable sur la jeunesse. À ce propos, Ch. Baudelaire dit: "Pour l'enfant amoureux de cartes et d'estampes, l'univers est égal à son vaste appétit.» (BAUdelaIRE, 1958: 278). 
Il s'ensuit que, à l'époque considérée, les ouvrages pour les enfants consacrés à la géographie constituent une part non négligeable de la littérature pour la jeunesse. La consultation de bibliographies et d'autres documents de référence le confirme. Hors des cercles de la bibliophilie et des chercheurs en histoire du livre d'enfant, peu de gens le savent. Il nous a paru donc intéressant d'évoquer l'existence de ce type d'ouvrages à cette époque-là.

\section{BIBLIOGRAPHIE 3}

s. a. (1783) - Atlas ecclésiastique, littéraire, civil, politique, militaire et commerçant de la France et du globe ou étrennes portatives, utiles et agréables pour l'année bissextile 1784. Beauvais et Froullé, Paris.

s. a. (1800) - Abrégé de géographie à l'usage de la jeunesse. Reuner, Neuveville.

s. a. (1812) - Abécédaire géographique,. Eymery, Paris.

s. a. (s. d. [vers 1815]) - Abécédaire géographique, Geographisches ABC und Lesebuch, Kollmann, Leipzig.

s. a. (1825) - Géographie (La) en estampes. Blanchard, Paris.

s. a. (1830) - Cours de géographie ancienne et moderne, suivi des élémens de l'astronomie. Piller, Fribourg en Suisse.

s. a. (1838) - Leçons de géographie à l'usage de l'enfance. Société reproductive des bons livres, Paris.

s. a. (1845) - Petite géographie vivante. Courcier, Paris.

s. a. (1979) - Encyclopaedia Universalis, volume 13: 377-397. Encyclopaedia Universalis, Paris.

Bailleul, J.-CH. et al. (1826) - Bibliomappe ou livre-cartes; leçons méthodiques de géographie et de chronologie, volume 10, Renard, Paris.

Baudelaire, CH. (1958) - Les fleurs du mal, Brocéliande, Strasbourg.

Bettencourt Pires, M. L. [vers 1983] - História da literatura infantil portuguesa, Vega, Lisboa.

Bourdon, C. (1744) - Elemens de géographie, ou nouvelle méthode simple et abrégée pour apprendre en peu de tems et sans peine la géographie. Barrau, La Haye.

BUfFIER, C. (1736) - Géographie universelle exposée dans les diférentes métodes qui peuvent abréger l'étude \& faciliter l'usage de cette sience. Giffart, Paris.

Buisson, F. (1968) - Répertoire des ouvrages pédagogiques du XVIe siècle. De Graaf, Nieuwkoop.

Chaix, P., Dufour, A., Moeckli, G. (1966) - Les Livres imprimés à Genève de 1550 à 1600. Droz, Genève.

Cinno, A. M. (1795) - Ristretto di geografia antica e moderna per i fanciulli con un trattato della sfera armillare. Giaccio, Napoli.

Della Bella, S. (1645) - Géographie. Le Gras, Paris.

\footnotetext{
3 Dans la bibliographie, «s. a.» signifie que les livres sont anonymes, «s. d.» signifie «sans date»; entre crochets figurent des informations qui n'apparaissent pas sur la page de titre mais que nous avons trouvées ailleurs.
} 
Gibrat, J.-P. (1768) - Traité de la géographie moderne, à l'usage des collèges et des pensions. Dalles, Toulouse.

Goldsmith, J. (1834) - A grammar of general geography, for the use of schools and young persons. Longman, Rees, Orme, Brown and Green, London.

Gordon, P. (1748) - Grammaire géographique ou analise exacte et courte du corps entier de la géographie moderne. Durand et Pissot, Paris.

Guichard, F. (1990) - Géographie du Portugal. Masson, Paris.

Huber, B. (1993) - «La géographie, un thème de prédilection ancien pour les auteurs de livres destinés à la jeunesse», Parole, $\mathrm{n}^{\circ}$ 26: 8-12.

Huber, B. (1998) - «L'enfant et la carte au XVIII' siècle», Mappemonde, nº 52: 35-38.

JAUFFRET, L.-F. (1830) - Géographie dramatique de la jeunesse ou nouvelle méthode amusante pour apprendre la géographie mise en dialogues et en scènes propres à être représentés dans les pensionnats et les familles. Maumus, Paris.

La Forest de Bourgon, [J. M.] (1709) - Alphabet géographique. Musier, Paris.

Legrand, A. (s. d. [vers 1825]) - Globe artificiel à l'usage du petit géographe. Legrand, Paris.

Lenglet Dufresnoy, N (1783) - Géographie des enfans ou méthode abrégée de la géographie. Chambeau, Avignon.

Louis XV (1718) - Cours des principaux fleuves et rivières de l'Europe. Imprimerie du Cabinet de S. M., Paris.

Marques, H. Jr (1928) - Algumas achegas para uma bibliografia infantil. Oficinas graficas da Biblioteca nacional, Lisboa.

Mentelle, E. (1781) - Cosmographie élémentaire, divisée en parties astronomique et géographique. Chez l'auteur, Paris.

Ostervald, F.-S. (1813) - Cours de géographie historique, ancienne et moderne, tome premier. Vincent, Lausanne.

Pluche, A. (1764) - Concorde de la géographie des différens âges. Estienne, Paris.

Rocha, N. (1992) - Breve história da literatura para crianças em Portugal. Biblioteca Breve, Lisboa.

Rousseau, J.-J. (1969) - Emile ou de l'éducation. Gallimard, Paris.

Saintes, A. de (1841) - Géographie parlante. Eymery, Paris.

[Sepp, C.], (1783) - Atlas des enfans, ou nouvelle méthode pour apprendre la géographie. Bruyset, Lyon.

Verreaux, E. et J. (1832) - L'Océanie en estampes. Nepveu, Paris. 